\title{
L'equidistanza ironica tra comico e tragico nella Mandragola di Machiavelli
}

\author{
Fulvio Orsitto \\ California State University, Chico \\ orsitto@gmail.com \\ https://dx.doi.org/10.12795/futhark.2011.i06.10
}

\begin{abstract}
This article analyzes The Mandrake, a theater play written by Machiavelli which, on the one hand has many aspects in common with other writings by the Florentine author and, on the other, offers plenty of fresh and innovative nuances. Indeed, if this comedy deals with some of Machiavelli's archetypical elements (such as Fortune and Virtue), one should also consider that the narration unfolds on a background that is neither necessarily historical, nor tragic and not even merely comical. As a consequence, this essay intends to apply to The Mandrake a category that may seem foreign to Machiavelli's universe. A category developed centuries after the Florentine writer and influenced by authors and thinkers such as Pirandello and Bergson, but also by the likes of an erudite scholar and writer such as Umberto Eco. A category called 'irony', based on the definition offered by Vladimir Jankélévitch.

The purpose of this article is to emphasize how the peculiarities of the ironic process prove to be useful in shedding some light on some aspects of this play, which cannot be analyzed as thoroughly by the classic interpretations usually applied to Machiavelli's texts. In fact, the category of irony - being situated halfway between comedy and tragedy - offers new interpretations that allow us to fill the gap between the comical and the tragic aspects that characterize Machiavelli's The Mandrake.
\end{abstract}

Keywords: MACHIAVELLI - THE MANDRAKE - ITALIAN LITERATURE - THE CINQUECENTO - THEATER - POLITICS - COMEDY - TRAGEDY - IRONY JANKÉLÉVITCH - ITALIAN RENAISSANCE - FORTUNE - VIRTUE POLITICAL THEORY - CALLIMACO - LUCREZIA - LIGURIO - NICIA 
Abstract: II presente studio è dedicato alla Mandragola, escursione machiavelliana nel regno del teatro che mantiene degli innegabili legami con le altre creazioni di quest'autore, non esitando però a colorarsi di nuove sfumature. Infatti, se è pur vero che questa pièce teatrale presenta lo svolgimento di elementi classici come la virtù e la fortuna, è anche degno di nota il fatto che essa si svolga lungo uno sfondo che non può essere ricondotto appieno all'orizzonte storico, a quello tragico e neppure a quello puramente comico. Pertanto, questa ricerca si propone di applicare alla Mandragola una categoria apparentemente estranea all'universo machiavelliano. Una categoria indubbiamente posteriore al mondo dello scrittore fiorentino e che deve la sua esistenza all'influenza di autori e pensatori come Pirandello e Bergson, ma anche dell'apporto di studiosi come Umberto Eco. Una categoria definita dall'appellativo 'ironia' ed elaborata partendo dalla definizione espressa inizialmente da Vladimir Jankélévitch.

L'intenzione di chi scrive è quella di sottolineare quanto le peculiarità del processo ironico siano opportune nel fare luce su alcuni aspetti di quest'opera che non possono essere approfonditi in modo altrettanto appropriato dai classici schemi di lettura a cui, in genere, vengono sottoposti i testi dello scrittore fiorentino. L'ironia infatti, proprio in virtù della contemporanea presa d'atto ma anche della frantumazione della totalità destinale che la colloca a metà strada tra commedia e tragedia, può essere in grado di offrirci un orizzonte interpretativo che ci permette di colmare quel vuoto ermeneutico esistente proprio tra comico e tragico, e lungo il cui arco si svolge gran parte della narrazione della Mandragola.

Keywords: MACHIAVELLI - MANDRAGOLA - LETTERATURA ITALIANA CINQUECENTO - TEATRO - POLITICA - COMMEDIA - TRAGEDIA - IRONIA - JANKÉLÉVITCH - RINASCIMENTO - FORTUNA - VIRTÙ - TEORIA POLITICA - CALLIMACO - LUCREZIA - LIGURIO - NICIA

"In spite of all the critical literature that has been written about Machiavelli's Mandragola, there remain a number of interpretative questions that merit further discussion. The comedy has elicited a wide range of interpretations, with some views quite diverse from what might be termed the traditional reading of the play. By far the majority of the readings develop a hypothesis about the relationship between Machiavelli's excursion into the 
realm of theater and his more serious political and historical writings"'.

Come confermato da Barber in questa citazione, su Machiavelli "historico, comico, tragico" 2 sono stati versati fiumi di parole. Ciononostante, chi scrive ritiene che sia ancora possibile, nonchè opportuno, proporre degli approcci ai testi dello scrittore fiorentino che abbiano la possibilità di evolversi lungo strade ermeneutiche ancora poco esplorate. Il presente studio è dedicato alla Mandragola, escursione machiavelliana nel regno del teatro che da un lato mantiene degli innegabili legami con le altre creazioni di quest'autore, ${ }^{3}$ ma che dall'altro non esita a colorarsi di nuove sfumature. ${ }^{4}$ Infatti, se è pur vero

1 Joseph A. Barber, "The Irony of Lucrezia: Machiavelli's Donna di virtü" Studies in Philology 82:4 (1985): 450-459, 450.

${ }^{2}$ Come lui stesso si definì in una lettera diretta all'amico Guicciardini.

${ }^{3}$ A proposito della Mandragola un critico come Ridolfi - in Roberto Ridolfi, "Introduzione" La Mandragola di Niccolò Machiavelli (Firenze: Leo S. Olschki Editore, 1965): 6-50, p-10 afferma che "in codesta mistione di farsa e di dramma, fusa al gran fuoco della poesia, i sali fiorentineschi, i lazzi, le grasse facezie, si mescolano con effetti singolari, di un fascino inesprimibile, a qualche massima dei Discorsi e del Principe"; mentre Cagli - in Bruno Cagli, "La commedia e la invida natura umana" Machiavelli. Tutto il teatro (Torino: Einaudi, 1979): 7-14, 13 - arriva a sostenere che il pessimismo di Machiavelli induce l'autore "a dare, con la Mandragola, le stessi chiavi alla soluzione dei problemi privati, offerte al Principe per la soluzione dei problemi degli Stati”. Ė poi Mansfield - in Harvey C. Mansfield, "The Cuckold in Machiavelli's Mandragola" The Comedy and Tragedy of Machiavelli. Essays on the Literary Works (New Haven: Yale University Press, 2000): 1-29, 1 - a ribadire che "the Mandragola is a heavily politicized comedy, that both its jokes and its erotic passions are managed, hence stunted, for the sake of a political end".

${ }^{4}$ In questo caso è Davico Bonino - in Guido Davico Bonino, "Introduzione alla Mandragola di Niccolò Machiavelli" Teatro. Andria, Mandragola, Clizia (Torino: Einaudi, 1979): VII-LX, XII-XIII - a sottolineare come "il Machiavelli della Mandragola porta a livelli di raffinatezza inconsueta il procedimento adottato (non senza qualche incertezza e discontinuità, e certo con minore eleganza) dai commediografi che l'hanno appena preceduto (un Ariosto, un Bibbiena), cioè la "contaminazione». Non solo egli dispone, rispetto a costoro, di materiali assai più vari e ricchi di suggestione: ma li manipola con una volontà di travestimento altrimenti scaltra". La contaminazione induce quindi Machiavelli ad avvicinare il comico al tragico. È poi Sullivan, fra gli altri, a confermare - in Vickie B. Sullivan, "Introduction" The Comedy and Tragedy of Machiavelli. Essays on the Literary Works (New Haven: Yale University Press, 2000): IX-XXI, XII - l'evidenza di questo aspetto, aggiungendo che "Scholars have also found tragedy in Machiavelli's comedy, arguing that his Mandragola is a dark satire that is at base a tragedy". Un critico come Martinez - in Ronald Martinez, "The Pharmacy of Machiavelli: Roman Lucretia in Mandragola" Renaissance Drama 14 (1983): Futhark 6 (2011)

Orsitto, L'equiditanza ironica,

205-228

ISSN 1886-9300 
che questa pièce teatrale presenta lo svolgimento di elementi per cosi dire classici (come la virtù e la fortuna), ${ }^{5}$ risulta però anche degno di nota il fatto che essa si svolga lungo uno sfondo che non può essere ricondotto appieno all'orizzonte storico, a quello tragico e neppure a quello puramente comico. Pertanto, questa ricerca si propone di applicare alla Mandragola una categoria apparentemente estranea all'universo machiavelliano. Una categoria indubbiamente posteriore al mondo dello scrittore fiorentino e che deve la sua esistenza all'influenza di autori e pensatori come Pirandello e Bergson, ma anche dell'apporto di studiosi come Umberto Eco. Una categoria definita dall'appellativo 'ironia' ed elaborata partendo dalla definizione espressa inizialmente da Vladimir Jankélévitch.

L'intenzione di chi scrive è quella di sottolineare quanto le peculiarità del processo ironico siano opportune nel fare luce su alcuni aspetti di quest'opera che non possono essere approfonditi in modo altrettanto appropriato dai classici schemi di lettura a cui, in genere, vengono sottoposti i testi dello scrittore fiorentino. L'ironia infatti, proprio in virtù della contemporanea presa d'atto ma anche della frantumazione della totalità destinale che la colloca a metà strada tra commedia e tragedia, ${ }^{6}$ può essere in grado di offrirci un orizzonte interpretativo che ci permette di colmare quel vuoto ermeneutico esistente proprio tra comico e tragico, e lungo il cui arco si svolge gran parte della narrazione della Mandragola.

1-43 - si spinge poi ancora oltre, individuando come fonte d'ispirazione della Lucrezia machiavelliana un episodio assolutamente tragico, quello occorso alla matrona romana Lucretia, delle cui virtù e del cui stupro Livio fornisce un ampio resoconto.

${ }^{5}$ Elementi assai cari allo scrittore fiorentino il quale, anche in questo caso, riesce a svolgerli in un'ambientazione che rivela, almeno in parte, dei richiami storici.

${ }^{6}$ Questa posizione peculiare è dovuta all'essenza stessa del procedimento ironico che lungi dall'essere neutro, si propone in tutto il suo essere - letteralmente - tragicomico. É infatti proprio grazie alla mescolanza di toni dolci ed amari, ed al caratteristico gioco di luci ed ombre tipico dell'ironia, che l'ironista riesce ad ottenere, grazie all'uso di un singolare chiaroscuro, un eccezionale effetto di rilievo a tutto tondo. In altre parole questa mescolanza di Apparire ed Essere, a cui possiamo attribuire il nome di ironia, si caratterizza per la sua capacità di graffiare significativamente con una risata amara che nasce dalla raggiunta presa d'atto del tragico, unita alla mai dimenticata consapevolezza del comico. 


\section{Analisi dei percorsi comico-tragico-ironici}

\section{Considerazioni sul comico}

È stato Aristotele uno dei primi pensatori a cercare di fornire una certa sistematicità al legame tra tragedia e comicità, ma purtroppo la parte finale della sua trattazione sul tragico, in cui approfondiva questi temi, è andata perduta. La stessa collocazione terminale di questo studio lascia però già intravedere, a detta di molti studiosi, un segno della minore considerazione in cui il filosofo greco teneva il comico rispetto al tragico. Umberto Eco, in particolare, sostiene che "per Aristotele il comico è qualcosa di sbagliato che si verifica quando in una sequenza di avvenimenti s'introduce un evento che altera l'ordine abituale dei fatti"7. Lo stesso Eco, in una delle intuizioni più brillanti contenute nella sua analisi sul comico, sottolinea l'essenziale natura ambigua e contraddittoria di quest'ultimo. II comico infatti, a detta dello studioso alessandrino, svolge sovente una funzione "di giustiziere, poiché ridimensiona uomini e istituzioni, demistifica e riveste i panni di una critica sociale," ${ }^{8}$ ma rimane altrettanto spesso uno "strumento di conservazione, poiché diverge le energie contestatarie ed acquieta le irritazioni”".

Beniamino Placido non sembra affatto allontanarsi da questa linea interpretativa e, nell'introduzione alla traduzione italiana de // riso di Bergson, pare spingersi addirittura oltre, arrivando a paragonare il comico al sogno, ${ }^{10}$ proprio in virtù della sua sostanziale forza di

\footnotetext{
7 Umberto Eco, Diario minimo (Milano: Edizioni Bompiani, 1992), 85. In seguito però Eco affermerà che questa inferiore lucidità analitica rispetto al tragico non impedisce alla risata ed al dileggio di abbattere perfino le costruzioni più seriose.

${ }^{8} \mathrm{Di}$ conseguenza il comico può anche giungere a mettere in dubbio i valori che la società ha condiviso fino ad un certo momento, avvalendosi della sua notevole forza nel demitizzare e nello sconsacrare. Da questa considerazione echiana emerge quindi una visione che dipinge il comico come uno strumento di rinnovamento linguistico e culturale, al punto che si potrebbe affermare che quelli che non si lasciano coinvolgere dal riso sono solo coloro che accettano dogmaticamente l'ordine prestabilito.

9 Umberto Eco, "II nemico dei filosofi." L'Espresso, 13 agosto 1967.

${ }^{10}$ II quale, anche a detta di Freud, è sempre conservatore, in quanto il suo scopo non è certo di disturbare il sonno ma di conservarlo.

Futhark 6 (2011)

Orsitto, L’equiditanza ironica,
}

205-228

ISSN 1886-9300 
conservazione dello status quo attraverso un'eversione che é solo momentanea. Lo stesso Bergson poi, nel saggio in questione, ${ }^{11}$ afferma che il riso è uno strumento di cui la società si serve per scoraggiare $\mathrm{e}$ penalizzare i comportamenti asociali, specie quelli statici e ripetitivi che interrompono la fluidità della vita sociale, la circolarità del suo svolgimento, la flessibilità della sua organizzazione, giungendo addirittura a sostenere che "questa rigidità è il comico, ed il riso ne è il castigo", ed infine che "il riso è veramente una specie di castigo sociale"12

Ciò che risalta con maggiore evidenza nel comico e nelle sue manifestazioni è il fatto che, tramite il riso, l'uomo riesca a vivere un'esperienza di regressione verso le sue funzioni più basilari, riducendosi ad una specie di marionetta. ${ }^{13}$ In secondo luogo la frantumazione della totalità destinale tipica del comico ci rammenta che un procedimento analogo viene utilizzato anche dall'ironia, ${ }^{14}$ preannunciandoci che sia l'approccio comico sia quello ironico nei confronti della realtà non sono altro che un anticipo di quell'abbraccio mortale con la totalità destinale che toccherà poi alla tragedia cercare di stringere.

Tragedia ed ironia

L'approccio tragico punta l'attenzione sul contenuto più che sulla forma. Pertanto, anziché avanzare un'analisi dell'esperienza umana

${ }^{11}$ A questo proposito si noti che si tratti di un'opera scritta agli inizi del secolo (1900) e quindi sostanzialmente coeva rispetto al saggio sull'umorismo di Pirandello; coincidenza che pare sottolineare come il passaggio del secolo abbia ispirato tutta una serie di riflessioni sul comico.

${ }^{12}$ Henry Bergson, Il riso. Saggio sul significato del comico (Bari: Ed. Laterza, 1987), 91.

${ }^{13}$ L'essenza profondamente umana del riso non nasconde poi neppure la presenza di legami atavici col satanico. Infatti, se da un lato la risata ci riconduce alle nostre funzioni più basilari e materiali, dall'altro, è proprio questo avvicinamento alla nostra parte più umana a far registrare un contemporaneo allontanamento dalla sfera più alta, quella del divino. Eco infatti, a questo proposito, cita Baudelaire sostenendo che "il riso è satanico ed è dunque profondamente umano" (Eco, Diario minimo, 264).

${ }^{14} \mathrm{La}$ quale però è in grado di abbinare alla succitata frantumazione della totalità destinale una, almeno parziale, presa d'atto della stessa che, inevitabilmente, sfugge ad un approccio esclusivamente comico. 
basata solo su parti di essa, si propone di affrontarla nella sua globalità. II risultato però non può che consistere in una resa di fronte all'incapacità di abbracciare l'incomprensibilità che pare governare le nostre vicende terrene, e nella conseguente rinuncia alla comprensione completa di un qualcosa che, comunque, sarebbe stato impossibile spiegare.

L'ironia, per contro, necessita di un parziale distacco dall'oggetto a cui intende riferirsi, in quanto l'ironista, a differenza del tragico, non aspira affatto ad essere profondo. Di conseguenza, la sua arte risulta più assimilabile all'apparire che all'essere, pur partendo comunque dallo stesso legame indissolubile con la realtà che l'autore tragico intende esporre e che egli, invece, cerca di criticare. Infatti, per sottolineare ulteriormente la presenza di questo contatto, potremmo arrivare addirittura a definire il sistema ironico proprio in base al suo fondarsi sullo "stringere qualcosa da una parte" afferrandolo e definendolo ${ }^{15}$ "tramite una connessione completa" 16 .

II procedimento ironico, pertanto, pare caratterizzarsi per una visione più precisa e dettagliata della realtà fenomenica e quindi per l'offerta di una via di fuga rispetto alla tragedia. ${ }^{17} \mathrm{Nel}$ momento in cui diventa automatismo, questo processo ci apre poi anche la possibilità di smascherare l'impeto dei sentimenti, rivelandocene spesso l'inconsistenza di fondo ed evitandoci di prendere in considerazione tutto dal punto di vista tragico. Ne consegue che l'ironia non risulti affatto impermeabile alle infiltrazioni del vissuto ${ }^{18}$ anzi, dato che é proprio da quest'ultimo che pare trarre la propria origine, si potrebbe addirittura affermare che il procedimento ironico corrisponda ad una vera e propria circonlocuzione della serietà.

\footnotetext{
${ }^{15}$ Un qualcosa che, ovviamente, non è che una piccola porzione di quella totalità destinale cui abbiamo già fatto cenno in precedenza.

${ }^{16}$ Vladimir Jankélévitch, L'ironia (Genova: Edizioni II Melangolo, 1987), 33.

${ }^{17}$ Ciononostante, se da un lato l'imminenza del processo ironico ci porta a concludere che ogni attimo, in sé e per sé, non meriti che un'attenzione divertita, dall'altro é l'insieme degli attimi (ossia la vita) a resistere alla forza del nostro humour, rivelandosi in tutta la sua tragicità.

${ }^{18}$ II quale nella sua totalità non può che essere visto come tragico (l'uomo sbaglia perché non é perfetto, l'uomo muore, etc.).

Futhark 6 (2011)

Orsitto, L'equiditanza ironica,
}

205-228

ISSN 1886-9300 
Riassumendo, si potrebbe quindi affermare che ironizzare significhi esprimere per velare, ma anche velare per meglio suggerire, ${ }^{19}$ dato che il processo ironico si caratterizza per il costante tentativo di convertire il suo destinatario verso ciò che si crede sia la verità mostrando - per usare un ossimoro - il visibile grazie all'invisibile. Alla luce di queste considerazioni è palese quindi la necessità di un meccanismo antifrastico all'interno del procedimento ironico, sebbene vada rilevato che l'importanza della netta inversione semantica che lo caratterizza non risiede tanto nel capovolgimento di senso di una parola, quanto piuttosto nel significato globale dell'enunciato. Infatti, nel gioco degli attanti della sequenza verbale ironica, ciò che conta è proprio la tendenza a valorizzare ciò che invece si intende svalorizzare. ${ }^{20}$

\section{II procedimento verbale ironico}

Alla luce di queste affermazioni il processo ironico si delinea quindi come un arabesco, grazie soprattutto alla sua capacità di travestirsi, di parlare per contrarium sfruttando un'arte la cui peculiarità è quella di non somigliare a se stessi e la cui arma essenziale risiede ovviamente nella nostra facoltà intellettiva. Infatti, pensando una cosa pur facendo il contrario, e distruggendo con una mano ciò che si è creato con l'altra, non facciamo altro che seguire un istinto profondamente umano, rimarcando come gli uomini non agiscano tanto in funzione

\footnotetext{
${ }^{19}$ Dato che si vuole comunicare per essere fraintesi pur trattandosi, alla fin fine, di un fraintendimento consapevole.

${ }^{20}$ Questa considerazione potrebbe forse erroneamente indurci a pensare ad una parentela con la menzogna ma, onde evitare un simile fraintendimento, è opportuno chiarire che tra ipocrisia ed ironia esiste la stessa differenza che passa tra la buona e la cattiva fede, tra uno stato di guerra ed uno stato di pace, tra una relazione unilaterale ed una bilaterale. Infatti non bisogna dimenticare che l'ironista stimola l'intellezione intessendo fra i due partner della comunicazione una complicità fatta di stima reciproca, mentre l'ipocrita instaura uno stato di passività subordinato ad uno di attività. L'origine del termine ipocrita,

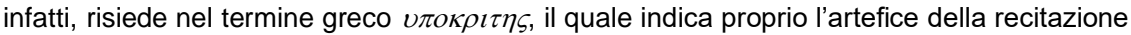

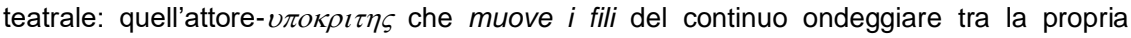
attività narcisistica e la passività voyeuristica del pubblico.
} 
dell'imminenza, quanto piuttosto delle conseguenze del loro comportamento presente. ${ }^{21}$

Secondo la concezione semiotica tradizionale, in ogni scambio comunicativo è possibile individuare la presenza di tre attanti: A1 (il parlante), A2 (il ricevente) ed A3 (la vittima). Nel caso specifico dell'ironia questi tre attanti si possono poi caricare di valenze ulteriori che rappresentano le sfumature dei ruoli che intendono ricoprire. In altre parole, si può definire A1 come l'ironista (colui che crea il messaggio ironico) mentre A2 si connota come il destinatario (l'interprete designato) ed A3, come il bersaglio, ossia la quintessenza del messaggio ironico stesso. Assai vario può essere, poi, il gioco di coincidenze tra questi tre ruoli. Infatti, si può verificare che A2 (l'interprete) ed A3 (la vittima) coincidano, oppure può avere luogo una situazione in cui A3 (la vittima) ignori del tutto il messaggio ironico, ed in questo caso si avrà una coincidenza tra A1 e A2. Alle volte invece può accadere che il parlante sia l'unico interprete del suo stesso messaggio, giungendo in alcuni casi anche all'autoironia che, a sua volta, potrà essere realizzata in maniera consapevole o meno.

Da tutto ciò consegue che l'ironista realizza quasi di continuo una sorta di attenuazione del proprio ego, facendo il possibile affinché il suo interlocutore provveda personalmente alla compensazione, mentre l'ironizzato, dal canto suo, non potrà fare a meno di ringraziarlo giungendo alla comprensione delle sue intenzioni. Un processo ironico pertanto non potrà che essere laconico, discontinuo ma, nel contempo, conciso, dato che - facendo affidamento sull'ascoltatore per sollevare il senso con la leva del segno - dovrà attribuire alla percezione il compito di completare con dei ricordi i segnali offerti dalla sensazione, preferendo essere significativo grazie ad uno stile ellittico piuttosto che completo per mezzo di uno enciclopedico. ${ }^{22}$ Ancora una volta, quindi, possiamo affermare che l'approccio ironico paia connotarsi sulla base di un procedimento circolare che viene invece negato a quello tragico a cui

${ }^{21} \mathrm{Si}$ potrebbe affermare che questo sia lo stesso istinto che spingeva $\mathrm{i}$ navigatori a navigare non per esporsi a dei pericoli ma in vista di un arricchimento, o che ci spinge talvolta ad ingerire una medicina amara certo non per soffrire, ma per guarire.

22 È fondamentale inoltre non fermarsi alla sola valutazione dell'aspetto aggressivo dell'ironia per considerare invece la sua dimensione comunitaria. 
potremmo, sostanzialmente, ascrivere invece tutto ciò che non ci è possibile abbracciare con la nostra comprensione.

Ironia e umorismo

Secondo Marina Mizzau l'ironia può inoltre trasformarsi in una sorta di critica sociale, diventando umorismo, "quando il meccanismo antifrastico riesce a sfruttare la situazione particolare per alludere, criticamente, a qualche sistema di valori generalizzato, raggiungendo il capovolgimento completo della comunicazione, delle convenzioni e degli stessi ruoli attribuiti”23. Pirandello, per contro, è piuttosto esplicito nello svalutare il procedimento ironico rispetto a quello umoristico, non esitando a relegare l'ironia al semplice livello di una "contraddizione puramente verbale, con connotazioni emotive limitate all'idea di un che di beffardo e mordace"24.

Bergson, invece, attribuisce all'ironia il compito di "enunciare quel che dovrebbe essere, fingendo di credere che esso sia precisamente ciò che è," permettendo quindi all'umorismo di dedicarsi a "descrivere minuziosamente e meticolosamente ciò che è, dando a credere che è proprio così che le cose dovrebbero essere" 25 . Potremmo pertanto concludere che il processo ironico come considerato da Bergson esprima l'esteriorità fingendo di prenderla per il reale, mentre la sua descrizione di umorismo miri a descrivere il reale simulando di scambiarlo per l'ideale. ${ }^{26}$

A questo punto però è opportuno avanzare ancora qualche ulteriore precisazione nei confronti della chiave di lettura della presente analisi. Infatti, oltre alle varie posizioni finora elencate, è opportuno dare spazio anche al punto di vista espresso da Jankélévitch, il quale ritiene che lo humour sia chiaramente una sottocategoria del procedimento ironico, collocandosi così in una posizione diametralmente opposta

\footnotetext{
${ }^{23}$ Marina Mizzau, L'ironia. La contraddizione consentita (Bologna: Ed. Feltrinelli, 1984), 40. ${ }^{24}$ Luigi Pirandello, "L'umorismo" I Grandi Classici (Novara: Ed. De Agostini, 1984), 26.

${ }^{25}$ Bergson, II riso, 110.

${ }^{26}$ Nella convinzione che 'fare dello humour' significhi ironizzare guardando lontano e - se possibile - ancora oltre, riconducendo in un secondo tempo la coscienza alla propria inafferrabile verità dopo un complesso cammino.
} 
rispetto a Pirandello. Nonostante gli studiosi sostanzialmente concordino nel sottolineare come entrambi i processi ci conducano a distruggere l'involucro esteriore delle istituzioni, è così necessario rimarcare le differenze esistenti tra questi due procedimenti. Più nello specifico, nell'analizzare la Mandragola è più efficace servirsi della proposta ermeneutica di Jankélévitch, proprio in virtù del fatto che quest'ultimo attribuisce una funzione meramente sociale all'umorismo rispetto all'ironia. ${ }^{27}$

Infine, è necessario ricordare che una delle discrepanze più significative emerse da una siffatta differenziazione tra tragico e comico risieda specificamente nella descrizione psicologica dei personaggi che, nel primo caso, si propone come cardine strutturale della vicenda, mentre nel secondo non può che essere semplicemente abbozzata. Infatti, è assolutamente evidente che il lettore non può che ridere del susseguirsi di varie peripezie e disgrazie, ma solo quando queste riguardano un personaggio a lui estraneo e lontano. A questo proposito, Bergson arriva a sostenere che il comico esiga, "per produrre tutto il suo effetto, qualcosa come un'anestesia momentanea del cuore" 28 . In altre

27 A questo proposito, per rimarcare ulteriormente la funzione sociale della categoria umoristica pirandelliana, è opportuno ricordare l'esempio fornitoci dallo stesso autore siciliano della donna che si imbelletta in maniera esagerata. Infatti, se ci limitiamo ad osservare questo personaggio femminile dall'esterno, ossia senza prendere coscienza delle motivazioni che possono averlo spinto ad un tale comportamento, siamo essenzialmente fermi ad un'analisi dell'aspetto comico. Se, invece, veniamo a conoscenza del fatto che lei ha assunto questo atteggiamento per cercare di piacere ancora a suo marito, abbiamo raggiunto, inevitabilmente, una dimensione gnoseologica più profonda, che Pirandello definisce come umorismo e che è caratterizzata da una risata amara, e non certo liberatoria come quella comica. Questa risata si carica infine di una forte valenza sociale, in quanto siamo più vicini al personaggio e ne viviamo con più partecipazione le vicende. A questo punto, come tassello conclusivo, si potrebbe anche proporre una piccola forzatura del pensiero pirandelliano, cioè un ulteriore avvicinamento alla vicenda destinale di un tale personaggio che, ipoteticamente, non è riuscito affatto a conquistare il consorte, il quale invece ha deciso di fuggire con una compagna molto più giovane. Ebbene, in questo caso, risulta evidente come la nostra maggiore compartecipazione rispetto al destino di questo personaggio, ormai carico di tonalità sempre più fosche, non possa che considerarsi come appartenente al mondo della tragedia, ossia a quel tentativo di approccio globale dell'esistenza umana che non può che risolversi con l'incapacità di abbracciare la terribile inspiegabilità che governa le nostre vicende terrene.

${ }^{28}$ Bergson, II riso, 6.

Futhark 6 (2011)

Orsitto, L'equiditanza ironica,

205-228

ISSN 1886-9300 
parole, ciò che pare emergere da queste considerazioni è che il maggior nemico del riso è proprio l'emozione.

La tragedia, per contro, si propone di infiammare e commuovere, si sforza di analizzare da vicino i personaggi per scavare nel loro animo, descriverne i travagli e giungere a far identificare lo spettatore con il protagonista portandolo a condividerne le sofferenze. ${ }^{29}$ Di conseguenza, quasi per non privarsi della risata, il comico tenderà sempre a rinunciare all'approfondimento della psicologia dei suoi personaggi.

Quando analizziamo il comico il nostro sguardo deve invece rivolgersi ad un altro registro. La comicità, infatti, rispetto all'ironia ed all'umorismo, non guarda altrove né simula alcunché e, non essendo allusiva, non arriva mai a pregnarsi d'intenzioni o di secondi fini ma giunge, al limite, ad operare una deflagrazione, una sorta di primo movimento spontaneo. Un importante punto in comune tra ironia e comicità risulta però essere la frantumazione della tragedia destinale, considerazione che ci porta a collocare l'ironista esattamente a metà strada tra il comico ed il tragico ${ }^{30}$ perché, da quella zona mediana del sorriso che lo ospita, spesso egli si arroga il titolo di punto di equidistanza tra 'positivo' e 'negativo' chiarendo, nel contempo, che la sua realtà non è il neutrum ma l'utrumque. ${ }^{31}$ Pertanto è proprio a quella mescolanza di apparire ed essere che è possibile attribuire l'appellativo di ironia, riconoscendole la capacità di graffiare significativamente con una risata amara che nasce dalla raggiunta presa d'atto del tragico unita alla mai dimenticata consapevolezza del comico.

In conclusione, se alla tragedia risulta possibile abbinare l'idea del pianto ed al comico quella del riso, non ci resta che accostare il procedimento ironico all'immagine di un sorriso, ma di un sorriso a scoppio ritardato (che presto sarà soffocato dalla consapevolezza) e che

\footnotetext{
${ }^{29}$ Pertanto lo spettatore vive in maniera differente l'intera vicenda e, senza rendersi conto che il comportamento di un Amleto può anche essere dissennato come quello di un miles gloriosus, patisce identificandosi con il primo, e ride (grazie al distacco) degli avvenimenti che turbano la vita del secondo.

${ }^{30}$ Per la sua costante capacità di evocare quel luogo di indifferenza a partire dal quale il riso tace senza che siano ancora spuntate le lacrime.

31 La sua essenza, infatti, non si propone come neutra ma, letteralmente, come tragicomica, e questo grazie alla mescolanza di dolce ed amaro in un caratteristico gioco di luci ed ombre che, con il suo chiaroscuro, ottiene un eccezionale effetto di rilievo a tutto tondo.
} 
si propone, a sua volta, come motus secundus, poiché può provocare una sottile risata pur non avendo voglia di ridere, e prendere in giro freddamente pur non desiderando divertirsi. La sua essenza si caratterizza per essere beffarda e cupa, ed il suo procedimento costante consiste nello scatenare una sorridente compiacenza per poi raggelarla immediatamente.

\section{La Weltanschauung dei personaggi della Mandragola}

\section{L'inganno di Lucrezia}

"Poi che l'astuzia tua, la sciocchezza del mio marito, la semplicità di mia madre e la tristizia del mio confessoro mi hanno condotta a fare quello che mai per me medesima arei fatto, io voglio iudicare che è venga da una celeste disposizione che abbi voluto così, e non sono sufficiente a recusare quello che 'I cielo vuole che io accetti” ${ }^{32}$.

Il primo personaggio che si delinea nel corso della lettura di questo testo machiavelliano non è affatto una delle varie creature di finzione che si alternano nel susseguirsi delle varie vicende, ma è l'autore stesso. Nel prologo, infatti, lo scrittore fiorentino ci propone un manifesto riferimento alla sua vita reale, non tanto per contestualizzare storicamente la narrazione, quanto per spiegare i motivi che lo hanno indotto a scrivere una pièce teatrale ${ }^{33} \mathrm{e}$, soprattutto, per rivelarci la chiave di lettura dell'intera opera nel momento in cui, rivolgendosi sia agli

32 Machiavelli, "La Mandragola", 133.

${ }^{33}$ Quanto Machiavelli soffrisse il fatto di essere costretto ad esprimersi tra le righe lo si può chiaramente intendere nel momento in cui ci confessa: "E se questa materia non è degna,/per esser pur leggieri,/d'un uom, che voglia parer saggio e grave,/scusatelo con questo, che s'ingegna/con questi van' pensieri/fare el suo tristo tempo più suave,/perchè altrove non have/dove voltare el viso,/chè gli è stato interciso/mostrar con altre imprese altra virtùe,/non sendo premio alle fatiche sue". Niccolò Machiavelli, "La Mandragola" Teatro. Andria, Mandragola, Clizia (Torino: Einaudi, 1979): 63-137, 68.

Futhark 6 (2011)

Orsitto, L'equiditanza ironica,

205-228

ISSN 1886-9300 
spettatori sia ai lettori, instaura un parallelo tra loro e quella "giovane accorta" di Lucrezia affermando: "lo vorrei che voi fussi ingannate come lei"34.

In questa frase è possibile ravvisare l'esordio di un procedimento circolare che si conclude nelle battute finali della commedia e che, proprio in virtù di questo accostamento tra i fruitori della narrazione e di quello che poi scopriremo esserne il perno, rivela la sua sostanziale forza, nonchè la sua essenziale coerenza rispetto al pensiero del Machiavelli più classico, ossia quello che emerge dalla pagine de II Principe. In altre parole, l'autore fiorentino non cessa neppure in questa commedia di dare consigli a coloro che si accostano alle sue opere, ${ }^{35}$ invitandoli a vivere l'esperienza di Lucrezia e, soprattutto, a reagire nel suo stesso modo, dimostrando cioè di sapersi avvalere di quel tipo di virtù che appartiene a quei pochi eccellenti principi che riescono ad affrontare la fortuna ed trarla a proprio vantaggio. ${ }^{36}$

Pertanto, proprio alla luce di queste considerazioni, risulta immediatamente chiaro come questo testo vada letto con profonda attenzione, per evitare di cadere nei tranelli interpretativi che il suo creatore ha sparso nel corso della narrazione. È evidente, infatti, che a prima vista si sia subito portati ad identificare la figura ideale del principe con quel Callimaco a cui Ligurio, sorta di alter ego machiavelliano all'interno dell'opera, non fa altro che dare consigli. ${ }^{37}$ Cionostante, da una lettura più accorta non può che emergere come la figura 'principe' della narrazione sia invece quella di Lucrezia la quale, sin dall'inizio, viene descritta come "atta a governare un regno" 38 . Secondo Barber,

${ }^{34}$ Machiavelli, "La Mandragola", 67.

${ }^{35}$ A detta di Paula Findlen la Mandragola "only tangentially referred to politics and instead offered sharp observations about life in a Renaissance city". Si veda Paula Findlen, "Understanding the Italian Renaissance" The Italian Renaissance (Malden: Blackwell Publishing, 2002): 4-40, 32.

${ }^{36}$ Ricorda ancora Findlen che "audiences laughed heartily at The Mandrake (1518), a tale of lust and deceit that subtly dissected the Florentine obsession with wealth and lineage, presenting the city as a world teeming with middlemen who knew how to close a deal". Si veda Findlen, "Understanding the Italian Renaissance", 32.

${ }^{37}$ Esattamente come lo scrittore fiorentino fa ripetutamente nel corso de II Principe.

38 A questo proposito vale la pena di ricordare come, nella sua descrizione offertaci da Callimaco, egli si soffermi non tanto sulle ricchezze del marito, quanto sul fatto che quest'ultimo "al tutto si lascia governare da lei". Machiavelli, "La Mandragola", 74. 
infatti, "it is clear from Callimaco's description that she is in complete control of herself, her family, and her social milieu"39. Di conseguenza, è proprio in questo apparente rovesciamento ermeneutico che si annida il nucleo centrale di una comunicazione ironica intesa nei termini in cui è stata descritta in precedenza. In buona sostanza, richiamando quel procedimento verbale a cui si è fatto cenno pocanzi, se si considera Machiavelli come il parlante-ironista (ossia A1) ed i lettori-spettatori come i riceventi-destinatari (cioè A2), non resta che leggere come A3 (quindi come messaggio di questo processo comunicativo) il capovolgimento stesso dell'orizzonte interpretativo.

L'ironia dell'autore, lungo questa prospettiva, si nutre del fatto che è proprio il personaggio in apparenza più debole, ossia quello che pare essere assolutamente in balia dei capricci di un giovane scioperato, ${ }^{40}$ a rivelarsi alla fine come quello maggiormente dotato di quella virtù tipicamente machiavelliana ${ }^{41}$ che permette di vincere le avversità della fortuna, riuscendo addirittura nell'impresa di volgerle a proprio vantaggio. In altre parole, l'inganno di Lucrezia è l'inganno da lei subito ma è anche, e soprattutto, un inganno che ella è in grado di ritorcere a proprio favore.

Successivamente, il fatto che lo scrittore fiorentino abbia deciso di rivolgersi direttamente agli spettatori ed ai lettori nel corso del prologo, augurandosi che possano vivere una simile esperienza, non può che portarci a credere che, anche in questo caso, il messaggio ironico sotteso ad una tale affermazione risieda ancora in un ulteriore rovesciamento di prospettiva. In pratica, attraverso questo auspicio, Machiavelli pare quasi comunicare ai fruitori della sua opera il suo desiderio che questo inganno si realizzi, ma in maniera consapevole. In questo modo una tale inversione semantica non fa che risolversi in un vero e proprio ammaestramento ad essere risoluti nel prendere le decisioni più opportune, anche quando queste sono in contrasto con la morale corrente e persino con i propri più intimi principi. ${ }^{42}$ Infatti, tutto ciò

\footnotetext{
${ }^{39}$ Barber, "The Irony of Lucrezia", 457.

${ }^{40}$ Nonchè dell'inettitudine del consorte ed, in generale, del profondo livello di corruzione della società.

${ }_{41}^{1}$ Una virtù che, in questo senso, potremmo chiamare anche machiavellica.

42 In questo senso la figura di Lucrezia ed, in particolare, la sua profonda connotazione religiosa non fanno che acuire il contrasto tra la morale in cui ella pare credere e la Futhark 6 (2011) Orsitto, L'equiditanza ironica,
}

205-228

ISSN 1886-9300 
si verifica grazie allo sviluppo del meccanismo antifrastico tipico del processo ironico, che affida la sua riuscita non tanto al netto capovolgimento del senso di una parola, quanto piuttosto ad un totale ribaltamento ermeneutico del significato dell'intero enunciato, ${ }^{43}$ secondo $\mathrm{i}$ dettami di quel gioco degli attanti della sequenza verbale ironica in cui ciò che conta è proprio la tendenza a valorizzare ciò che invece si intende svalorizzare. ${ }^{44}$

Come conseguenza, richiamandosi ancora a Barber, si potrebbe affermare che "the irony is that the traditional winner gets the girl but is the comedy's real loser, and vice-versa; once seduced, Lucrezia takes charge of the situation and emerges as a true donna di virtú" ${ }^{45}$. Infatti, proprio "through her own volition and through a positive mutazione she regains control" 46 , al punto che nel colloquio finale con messer Nicia all'accusa velata di essere un pò troppo ardita risponde: "Egli è la grazia vostra!"47

\section{Il gioco delle parti di Callimaco e Ligurio}

"Avendo compartito el tempo, parte alli studi, parte a' piaceri e parte alle faccende; e in modo mi travagliavo in

risolutezza delle sue azioni conclusive che, da personaggio in balia degli eventi, la trasformano in vero e proprio vincitore morale di una narrazione su cui dimostra di aver assunto un controllo pressochè totale.

${ }^{43} \dot{E}$ in questa accezione che, infatti, vanno lette le parole di Lucrezia quando si rivolge a Callimaco ed afferma: "Però io ti prendo per signore, padrone, guida; tu mio padre, tu mio defensore, e tu voglio che sia ogni mio bene; e quello che 'I mio marito ha voluto per una sera, voglio ch'egli abbia sempre." (V.iv)

44 A questo proposito, secondo una provocatoria interpretazione proposta da Antonio Gramsci, "le opere del Machiavelli sono di carattere «individualistico», espressioni di una personalità che vuole intervenire nella politica e nella storia del suo paese e in tal senso sono di origine "democratica»". Si veda Antonio Gramsci, Note sul Machiavelli, sulla politica e sullo Stato moderno (Roma: Editori Riuniti, 1971), 149.

${ }^{45}$ Barber, "The Irony of Lucrezia", 458.

${ }^{46}$ Barber, "The Irony of Lucrezia", 458.

${ }^{47}$ Secondo i dettami del classico gioco degli attanti del procedimento ironico che la vedono, in questo caso in qualità di $A 1$, ossia ironista, al cospetto del suo consorte Nicia, cioè $A 2$, il destinatario che è nel contempo anche bersaglio (in pratica $A 3$ ) di tutto questo processo comunicativo. 
ciascuna di queste cose, che l'una non mi impediva la via dell'altra. E per questo, come tu sai, vivevo quietissimamente, giovando a ciascuno e ingegnandomi di non offendere persona; tal che mi pareva esser grato a' borghesi, a gentiluomini, al forestiero, al terrazzano, al povero, al ricco"48.

Callimaco.

Ligurio.

Callimaco.

Ligurio.

Callimaco.

In contrapposizione alla succitata repentina presa del potere da parte della figura di Lucrezia va invece registrata la costante degradazione della figura di Callimaco, in particolare nei confronti del suo interlocutore "principe", quel Ligurio che dimostra ben presto di sapersi affrancare dal ruolo angusto di consigliere. Infatti, colui che nel prologo viene semplicemente definito come 'un parassito' e che lo stesso Callimaco descrive - durante un dialogo col famiglio Siro - come uno che "s'è dato a mendicare cene e desinari" 50 , ci mostra ben presto di possedere una consapevolezza che sfugge a molti personaggi in apparenza socialmente o culturalmente più elevati.

Già nel corso del primo atto Ligurio manifesta di non essere affatto un elemento secondario nella vicenda, ed il suo ruolo inizia così a trasformarsi lentamente da quello di mero esecutore di un disegno nefando a quello di indispensabile consigliere fraudolento. Del resto, nel momento stesso in cui assistiamo al suo primo dialogo con Callimaco ci rendiamo immediatamente conto che colui il quale, in un primo momento sembrava descritto con tutte le qualità tipiche del principe,${ }^{51}$ in realtà non

${ }^{48}$ Machiavelli, "La Mandragola", 72.

49 Machiavelli, "La Mandragola", 93.

${ }^{50}$ Machiavelli, "La Mandragola", 75.

51 Infatti, per quanto il suo perdersi nelle letture ci dia, da un lato, un chiaro riferimento allo stesso Machiavelli (quando nella lettera al Vettori del 10 dicembre 1513 descrive il suo trasferirsi in una humanità di cui si nutre attraverso la lettura dei classici), dall'altro sembra Futhark 6 (2011)

Orsitto, L'equiditanza ironica,
"Tu mi pari cotto."
205-228

ISSN 1886-9300 
è altro che un personaggio debole, che per comprendere appieno la realtà che lo circonda ha bisogno di avvalersi di quel filtro interpretativo che solo Ligurio è in grado di fornirgli. ${ }^{52} \mathrm{Di}$ conseguenza, questo inconsueto saggio parassita ha la non comune opportunità di dimostrarsi incredibilmente abile nel fornire sempre l'interpretazione migliore, e nel dispensare tutti i consigli indispensabili per raggiungere gli scopi prefissati dal suo debole principe. ${ }^{53}$

Risulta pertanto inevitabile, a questo punto, rilevare un parallelo tra la figura di Ligurio e quella dello stesso Machiavelli, entrambi personaggi in grado di riuscire, in qualche modo, a liberarsi dall'aura di figure secondarie per ascendere a ruoli di una rilevante importanza organizzativa, certamente più consona alle loro doti di consiglieri virtuosi. ${ }^{54}$ Ligurio infatti, in qualità di profondo conoscitore nonchè di esperto manipolatore delle cose umane, si presenta costantemente anche come una sorta di deus ex machina in grado di tirare i fili di tutta la vicenda senza neppure preoccuparsi di avvertire gli altri personaggi di quello che egli ha in mente per loro. ${ }^{55}$

forse ancora più rilevante notare come questa figura corrisponda in realtà a quella di un principe, ma non certo a quella del condottiero invocato dallo scrittore fiorentino, quanto piuttosto all'immagine di un principe debole, nient'affatto conscio nè tanto meno inserito nella specificità storica che lo circonda.

${ }^{52} \mathrm{~A}$ questo proposito è assolutamente emblematico il dialogo in cui è proprio Ligurio ad indicare a Callimaco la pericolosità insita nel cercare di fare breccia nel cuore di Lucrezia in un ambiente come quello dei bagni. Egli, infatti, dimostra una eccellente lucidità interpretativa, (per contro, totalmente assente in Callimaco) non mancando di rimarcare come in questa situazione "potrebbe venirvi uomo a chi madonna Lucrezia piacessi come a te, che fussi più ricco di te, ch'avessi più grazia di te". Machiavelli, "La Mandragola", 79.

${ }^{53}$ Naturalmente, è proprio Ligurio a proporre il tranello che permetterà a Callimaco di farsi passare per dottore, nonchè ad escogitare gli accorgimenti che, successivamente, faranno apparire quest'ultimo come uno sconosciuto, la perfetta vittima sacrificale in grado di soddisfare il morboso desiderio di messer Nicia di avere un erede.

${ }^{54}$ Ovviamente, in questo caso, nel senso più machiavellico del termine.

${ }^{55}$ Nella fattispecie, un esempio mirabile è fornito dal dialogo tra Ligurio, Callimaco e Nicia all'inizio dell'atto secondo in cui Ligurio lo stratega dimostra tutta la sua padronanza della situazione nel guidare il dialogo tra gli altri due personaggi lungo binari che, sia Nicia sia lo stesso Callimaco, non si aspettavano affatto. In questo senso potremmo poi affermare che questo personaggio rappresenti quasi la proiezione inconscia del desiderio che Machiavelli aveva di intervenire in maniera effettuale sulla società del suo tempo; desiderio che, suo malgrado, rimarrà in ampia misura irrealizzato. 
La profonda conoscenza che Ligurio ha del mondo si manifesta in una lunga serie di affermazioni che possiamo ricondurre ad uno schema di tipo ironico. Tra queste spicca, per esempio, la sua descrizione di come sia possibile, a livello pratico, disporre della collaborazione di un religioso come fra Timoteo per la realizzazione degli obiettivi di Callimaco. Peraltro nella frase: "lo, e danari, la cattiveria nostra, loro"56 è difficile non sentire riecheggiare l'eco dei discorsi machiavelliani al principe ideale; parole che, anche in quel caso, nascono da una profonda conoscenza della realtà effettuale.

Procedendo più nello specifico, la natura profondamente ironica di questa affermazione di Ligurio risiede nel fatto che, all'apparenza, essa è rivolta solo ai suoi due uditori, Nicia e Callimaco ma in pratica attraverso il consueto rovesciamento semantico - risulta indirizzata ancora una volta a tutti i fruitori del testo machiavelliano. Ligurio infatti, nel suo dialogo avvia un procedimento ironico che, lungi dall'essere afferrato dal suo ricevente primario A2, dichiara implicitamente di porsi come destinatario ancora più profondo quell'umanità varia che, nel corso del tempo, si è abbeverata alla fonte machiavelliana. ${ }^{57}$ Questo destinatario implicito corrisponde poi anche al significato più profondo dello scambio comunicativo ironico, ossia ad un messaggio che, in apparenza, è rivolto solo ai protagonisti della vicenda ma che, in realtà, tocca la totalità destinale di tutti gli esseri umani, perchè in questo caso è tutta l'umanità ad essere destinataria di questo scambio comunicativo.

Un simile gioco di attanti ci viene poi riproposto anche nel terzo atto, quando Ligurio, rivolgendosi ancora una volta a messer Nicia afferma che "fra Timoteo è per fare ogni cosa"58. Ebbene, anche in questa situazione l'attante $A 1$, ossia l'ironista, ${ }^{59}$ ha come destinatario $A 2$, Nicia, e come messaggio dello scambio comunicativo che intende proporre $A 3$, cioè un messaggio ironico che, anche in questo caso, riguarda proprio il destinatario a cui si sta rivolgendo. In pratica, anche in

${ }^{56}$ Machiavelli, "La Mandragola", 92.

57 Esattamente come lo scrittore fiorentino era solito dissetarsi grazie alla sapienza dei classici. Come ricorda ancora Findlen, infatti, per Machiavelli si trattava di "a secular ritual in which he entered the courts of ancient men to commune with their wisdom". Si veda Findlen, "Introduction to Part V. The Power of Knowledge", 216.

${ }^{58}$ Machiavelli, "La Mandragola", 104.

${ }^{59}$ Figura che, come abbiamo visto corrisponde a Ligurio ma richiama lo stesso Machiavelli. Futhark 6 (2011) Orsitto, L'equiditanza ironica,

205-228

ISSN $1886-9300$ 
questo caso, il meccanismo antifrastico pur essendo dedicato in apparenza a frà Timoteo intende rimarcare come, in realtà, il personaggio disposto a fare qualsiasi cosa sia proprio il marito di Lucrezia. ${ }^{60}$

La situazione di maggiore degrado è però quella vissuta dal personaggio di Callimaco. Quest'ultimo infatti, a differenza di Nicia che viene sempre descritto tramite una rappresentazione al limite dello slapstick, ${ }^{61}$ parte da un livello di profonda nobiltà - almeno apparente creato dall'immediato accostamento con l'otium culturale a cui si era abbandonato durante il soggiorno parigino, per poi cedere ad un progressivo abbrutimento morale ed anche fisico. ${ }^{62}$

Alla continua perdita di controllo della situazione registrata da parte di Callimaco fa invece riscontro una evidente acquisizione di potere da parte di Ligurio il quale, in breve, arriva a prendersi gioco di colui da cui era stato inizialmente ingaggiato ${ }^{63}$ ed a dettare ordini agli altri personaggi. ${ }^{64}$ Cionostante, anche a Callimaco viene lasciata la possibilità di esprimersi secondo un registro ironico, secondo l'ormai classico gioco degli attanti. Nella fattispecie, ció si verifica quando, nel

${ }^{60} \mathrm{Ma}$, anche in questo caso, A2 non coglie l'ironia.

61 Rappresentazione che però, in alcune situazioni, non esita a colorarsi di sfumature umoristiche nel senso pirandelliano del termine (soprattutto nei momenti in cui Machiavelli indugia maggiormente sul fatto che, tutto sommato, Nicia faccia tutto ciò in quanto accecato dal desiderio di avere un erede a tutti i costi ).

62 È significativo, a questo proposito, il fatto che Ligurio quasi ordini a quello che, almeno all'inizio, era raffigurato come il suo signore, di non mascherarsi nel corso della notte in cui avrebbe dovuto essere l'oggetto del rapimento ad opera sua e di Nicia. Ė poi altrettanto interessante notare come la spiegazione ufficiale consista nell'intenzione di non farsi riconoscere, mentre quella reale, il cosiddetto $\mathrm{A} 3$, risieda ancora una volta nel prendersi gioco di A2 (in questo caso Callimaco), a cui viene chiesto di storcere il viso, aprire, aguzzare e digrignare la bocca, nonchè chiudere un occhio.

${ }_{63}$ Come rimarcato in precedenza e come ribadito ulteriormente durante la scena del rapimento di Callimaco, Ligurio, con la scusa di una presunta veridicità, arriva persino a rivolgersi a quest'ultimo dicendo: "Via, ribaldo, tira via! S'i' ti sento favellare, io ti taglierò el collo!" Machiavelli, "La Mandragola", 129.

64 Infatti, quando non è Ligurio a dare ordini in maniera diretta è lo stesso Callimaco a pensarci, ed a rivolgersi al suo famiglio per raccomandargli: "Tu hai questa sera a fare tutto quello che ti dirà Ligurio; e fa conto, quando e' ti comanda, che sia io; e ciò che tu vedi, senti o odi, hai a tuo tenere secretissimo, per quanto tu stimi la roba, l'onore, la vita mia e il bene tuo". Machiavelli, "La Mandragola", 119. 
punto più estremo della sua perdita di controllo sugli avvenimenti, ${ }^{65}$ in qualità di ironista (A1) si rivolge a messer Nicia (A2) per dirgli: "venir vi possa el diavolo allo letto, dapoi ch'io non vi posso venir io!"66

\section{Conclusioni}

La scelta dell'ironia come chiave di lettura della Mandragola e dell'evoluzione di alcuni dei personaggi dell'opera ha rivelato, nel corso del presente studio, un suo carattere di sostanziale peculiarità e, allo stesso tempo, di contingente adeguatezza. Infatti, nel mettere in rilievo alcuni aspetti altrimenti difficilmente spiegabili in base a categorie ermeneutiche classiche, ${ }^{67}$ questo tipo di approccio ${ }^{68}$ ha dimostrato di riuscire a fornire interessanti rilievi sugli sviluppi di figure come quella di Ligurio, Callimaco e, soprattutto, Lucrezia.

Di conseguenza, chi scrive ritiene sia giusto sostenere che l'ironia abbia mostrato la sua piena applicabilità nei confronti di questo testo, al punto che potrebbe persino rivelarsi arduo scoprire altre chiavi di lettura in grado di spiegare in modo altrettanto soddisfacente personaggi e situazioni non chiaramente riconducibili ad un'ottica prettamente comica, tragica o storica. ${ }^{69}$ Un esempio in questo senso ci viene fornito dall'uso abbondante del soliloquio. Questo artificio retorico, infatti, ci avvicina molto allo sviluppo interiore dei protagonisti della narrazione e si richiama idealmente all'idea di un orizzonte tragico, in quanto ci permette di seguire più da vicino la loro evoluzione psicologica. Ciononostante, il ricorrere di questa cifra stilistica non garantisce affatto uno sviluppo consono ad una tragedia, riuscendo al massimo a farci prendere le distanze da una prospettiva solo ed esclusivamente comica.

65 Ossia nel momento in cui viene fatto oggetto del rapimento tramite il quale verrà introdotto nel talamo di Lucrezia dal suo stesso marito.

${ }^{66}$ Machiavelli, "La Mandragola", 125.

67 Come ad esempio la prospettiva storica, quella comica, quella tragica, oppure quella dell'umorismo pirandelliano.

${ }^{68}$ Che si basa sulla collocazione dellironia in uno spazio intermedio rispetto al processo comico ed a quello tragico.

${ }^{69}$ A differenza di altre opere machiavelliane.

Futhark 6 (2011)

Orsitto, L’equiditanza ironica,

205-228

ISSN 1886-9300 
Per quanto concerne l'orizzonte del riso, va poi sottolineato come quest'ultimo domini solo in apparenza la maggioranza dei personaggi. Infatti molte figure - specialmente Nicia - se da un lato si delineano spesso secondo canoni quasi slapstick, dall'altro non si limitano mai a quel ridicolo che tutt'al più sfiorano, richiamandoci sempre alla necessità di un codice interpretativo più complesso, come quello fornitoci dall'ironia.

È pertanto alla luce di queste considerazioni, nonchè della necessità di una approccio più onnicomprensivo, che l'applicazione della categoria ironica si adatta bene a questa opera. Del resto, personaggi come Callimaco, Ligurio e persino lo stesso Nicia, non si possono spiegare solo in un'ottica comica, ma neanche alla luce di una lettura tragica, o tanto meno in chiave storica. La stessa Lucrezia poi non è certo una delle tante marionette che si agitano nelle commedie, anche se non arriva neppure a connotarsi della tragicità di chi si appresta ad affrontare la crudeltà del fato. La sua è, infatti, una figura ironica, capace di oscillare tra la frantumazione ed il tentativo di comprendere quella tragedia destinale che la fortuna le ha dato in sorte ed in grado, al momento opportuno, di sfoggiare un'impensabile dose di machiavellica virtù.

In conclusione, è opportuno rimarcare ancora una volta come sia stata proprio la dissacrante prospettiva offerta dall'ironia ad aver reso possibile l'apertura e lo sviluppo di un orizzonte ermeneutico nuovo, lungo il quale "it becomes apparent as the play progresses that the successful seducer, Callimaco, becomes a more and more fitting example of one of life's foolish failures. Ironically the woman seduced, Lucrezia, emerges as a true embodiment of Machiavelli donna di virtú"70. È stata infatti proprio la versatilità di questo processo ad aver fornito una nuova preziosa chiave interpretativa che, grazie all'intrinseco rovesciamento semantico, ci permette di scoprire la forza e l'importanza di personaggi in apparenza perdenti, nonchè il fallimento di personaggi in apparenza vincenti.

Fulvio Orsitto, California State University, Chico

${ }^{70}$ Barber, "The Irony of Lucrezia", 450. 
RIFERIMENTI BIBLIOGRAFICI

BARBER, Joseph A. "The Irony of Lucrezia: Machiavelli's Donna di virtù" Studies in

Philology 82:4 (1985): 450-459.

BERGSON, Henry. Il riso. Saggio sul significato del comico. Bari: Ed. Laterza, 1987.

CAGLI, Bruno. "La commedia e la invida natura umana" Machiavelli. Tutto il teatro.

Torino: Einaudi, 1979, 7-14.

DAVICO BONINO, Guido. "Introduzione alla Mandragola di Niccolò Machiavelli" Teatro.

Andria, Mandragola, Clizia. Torino: Einaudi, 1979, VII-LX. ECO, Umberto. "II nemico dei filosofi." L'Espresso, 13 agosto 1967.

---. Diario minimo. Milano: Edizioni Bompiani, 1992.

FINDLEN, Paula. The Italian Renaissance. Malden: Blackwell

Publishing, 2002.

GRAMSCI, Antonio. Note sul Machiavelli, sulla politica e sullo Stato moderno. Roma:

Editori Riuniti, 1971.

JANKÉLÉVITCH, Vladimir. L'ironia. Edizioni II Melangolo, 1987.

MACHIAVELLI, Niccolò. "La Mandragola" Teatro. Andria, Mandragola, Clizia. Torino:

Einaudi, 1979, 63-137.

MANSFIELD, Harvey C. "The Cuckold in Machiavelli's Mandragola" The Comedy and

Haven: Yale

Tragedy of Machiavelli. Essays on the Literary Works. New

University Press, 2000, 1-29.

MARTINEZ, Ronald. "The Pharmacy of Machiavelli: Roman Lucretia in Mandragola"

Renaissance Drama 14 (1983): 1-43.

MIZZAU, Marina. L'ironia. La contraddizione consentita. Bologna: Ed. Feltrinelli, 1984.

PIRANDELLO, Luigi. "L'umorismo" I Grandi Classici. Novara: Ed. De

Futhark 6 (2011)

Orsitto, L'equiditanza ironica,

205-228

ISSN 1886-9300 
Agostini, 1984.

RIDOLFI, Roberto. "Introduzione" La Mandragola di Niccolò Machiavelli. Firenze: Leo S.

Olschki Editore, 1965, 6-50.

SULLIVAN, Vickie B. "Introduction" The Comedy and Tragedy of Machiavelli. Essays on $\mathrm{XXI}$.

the Literary Works. New Haven: Yale University Press, 2000, IX- 